\title{
Resolving the kpc jet of Centaurus A in TeV gamma-rays
}

\section{M. de Naurois ${ }^{* a}$, M. Holler ${ }^{b}$, Frank Rieger ${ }^{c}$, D. Sanchez ${ }^{d}$ and Andrew Taylor ${ }^{e}$ on} behalf of the H.E.S.S. Collaboration ${ }^{\dagger}$

${ }^{a}$ Laboratoire Leprince-Ringuet, École Polytechnique, CNRS, Institut Polytechnique de Paris, F-91128 Palaiseau, France

${ }^{b}$ Institut für Astro- und Teilchenphysik, Leopold-Franzens-Universität Innsbruck, A-6020 Innsbruck, Austria

${ }^{c}$ Max-Planck-Institut für Kernphysik, P.O. Box 103980, D 69029 Heidelberg, Germany

${ }^{d}$ Laboratoire d'Annecy de Physique des Particules, Univ. Grenoble Alpes, Univ. Savoie Mont Blanc, CNRS, LAPP, 74000 Annecy, France

${ }^{e}$ DESY, D-15738 Zeuthen, Germany

E-mail: contact.hess@hess-experiment.eu

\begin{abstract}
The point-spread function (PSF) of existing Imaging Atmospheric Cherenkov Telescopes is limited to scales of a few arcminutes. Measuring source sizes at or below the PSF level is generally restricted by statistics as well as the systematic uncertainties of the PSF characterisation. Employing new simulation and analysis techniques, the understanding of the PSF of the H.E.S.S. array has considerably increased, allowing to measure extensions of very high energy (VHE; E > $100 \mathrm{GeV}$ ) gamma-ray sources down to below one arcminute. Here we present the first detection of extended VHE emission from an extragalactic source, the kpc jet of the nearby radio galaxy Centaurus A. The physical extension of the VHE jet of Centaurus A exceeds $1.8 \mathrm{kpc}$, meaning that a large part of the emission originates far away from the central black hole. Our results as well as physical implications are going to be discussed.
\end{abstract}

36th International Cosmic Ray Conference -ICRC2019-

July 24th - August 1st, 2019

Madison, WI, U.S.A.

\footnotetext{
* Speaker.

${ }^{\dagger}$ for collaboration list see PoS(ICRC2019)1177
} 


\section{Acknowledgments}

See https: / / www.mpi-hd.mpg.de/hfm/HESS/pages/publications/auxiliary/ HESS-Acknowledgements-2019.html. 